Louisiana State University

LSU Digital Commons

Faculty Publications

Department of Mathematics

$9-1-2018$

\title{
Effective Maxwell's Equations for Perfectly Conducting Split Ring Resonators
}

Robert Lipton

Louisiana State University

Ben Schweizer

Technische Universität Dortmund

Follow this and additional works at: https://digitalcommons.Isu.edu/mathematics_pubs

\section{Recommended Citation}

Lipton, R., \& Schweizer, B. (2018). Effective Maxwell's Equations for Perfectly Conducting Split Ring Resonators. Archive for Rational Mechanics and Analysis, 229 (3), 1197-1221. https://doi.org/10.1007/ s00205-018-1237-1

This Article is brought to you for free and open access by the Department of Mathematics at LSU Digital Commons. It has been accepted for inclusion in Faculty Publications by an authorized administrator of LSU Digital Commons. For more information, please contact ir@lsu.edu. 
Effective Maxwell's equations for perfectly conducting split ring resonators

\author{
Robert Lipton, Ben Schweizer
}




\title{
Effective Maxwell's equations for perfectly conducting split ring resonators
}

\author{
Robert Lipton $^{1}$ and Ben Schweizer ${ }^{2}$
}

December 19, 2016

\begin{abstract}
We analyze the time harmonic Maxwell's equations in a geometry containing perfectly conducting split rings. We derive the homogenization limit in which the typical size $\eta$ of the rings tends to zero. The split rings act as resonators and the assembly can act, effectively, as a magnetically active material. The frequency dependent effective permeability of the medium can be large and/or negative.
\end{abstract}

MSC: 35B27, 78M40, 35B34

\section{Introduction}

Meta-materials consist of a large number of small elements. While each of the elements contains only ordinary materials, the properties of the meta-material can be very different from those of its constituents. In the theory of light, the index of refraction of every ordinary material is positive, but today we know how to construct meta-materials that have, effectively, a negative index of refraction. The astonishing properties of negative index materials has been investigated already in 1968 by Veselago [30], the first ideas how to construct a negative index meta-material appeared around 2000, an experimental realization of such a material was reported in [29]. In the subsequent years, many possible applications have been investigated, e.g. perfect lensing [24], directing light-beams around obstacles [25], or cloaking by anomalous localized resonance $[17,22]$.

The first ideas for negative index meta-materials were based on constructions with split rings. In these constructions the ring serves as an inductive element and the slit serves as a conductive element. The combination of the two elements forms an oscillator. When the oscillator (which is smaller than the

\footnotetext{
${ }^{1}$ Louisiana State University, Department of Mathematics, 384 Lockett Hall, Baton Rouge, U.S.A.

${ }^{2}$ TU Dortmund, Fakultät für Mathematik, Vogelpothsweg 87, D-44227 Dortmund, Germany.
} 
wave-length of light) is in resonance with the frequency of the light, resonance effects can lead to large local fields. When this is the case, the macroscopic features of the meta-material are given by effective coefficients (permittivity and permeability) that can be very different from the coefficients of the materials that are used in the construction: they can be very large or they can have the opposite sign. For a recent general overview regarding the effect of resonances in many small resonators see [27].

The mathematical analysis of negative index meta-materials is possible with the methods of homogenization theory, which has roots in the 1970s [3]. Until today, the most powerful tool for the homogenization of periodic problems is the method of two-scale convergence [1]. The homogenization of Maxwell's equations was already performed in [15], but the analysis of homogenization settings that lead to unexpected effective equations was pioneered by Bouchitté. Together with co-authors, he derived effective systems with negative effective permittivity [14], with nonlocal effects [6], and with an effective magnetic response [5]. The three-dimensional Maxwell's equations have been analyzed first in [7] using the split ring geometry, later in [4] with dielectric Mie-resonators, and in [19] with flat rings. Very recently, also the combination of resonator elements with wire elements was successfully analyzed. The setting is very close to the experimental set-up of the early negative index constructions; the mathematical analysis reveals that it is possible to obtain an effective system with two coefficients $\varepsilon_{\text {eff }}$ and $\mu_{\text {eff }}$, which have both a negative real part [21]. With that contribution, we have a mathematical confirmation and an effective description of a negative index meta-material.

The above contributions regard results in the language of two-scale homogenization results, i.e. the derivation and justification of effective equations. For other approaches using local resonances see [18, 23]. A recent development focuses on negative index metamaterials generated by subwavelength plasmon resonances associated with noble metals in the optical regime see $[9,10,11,12]$. There, frequency intervals are identified over which negative group velocity and power flow occur for coated inclusions and dispersed phases.

Regarding new ideas for the experimental design of negative index materials see e.g. [16, 28]. Apart from constructing negative index materials (with all the possible applications of these materials), there are other examples where homogenization leads to unexpected effective equations, describing astonishing effects: Resonance with surface plasmons can induce perfect transmission through sub-wavelength holes [8]. The resonance in small Helmholtz resonators can lead to dispersive effects in the propagation of sound waves [20].

The results of this contribution. We consider a split ring geometry with perfectly conducting materials. The latter means that the metal inclusion is not described by a large permittivity; instead, the electric and the magnetic field vanish inside the metal and the metal interacts only via boundary conditions with the fields. We perform the homogenization limit, i.e.: We consider solutions to the time-harmonic Maxwell's equations and study their limiting 
behavior as $\eta \rightarrow 0$, where $\eta>0$ is the typical size of the single split ring. We obtain that the limiting fields are described by time-harmonic Maxwell's equations with effective parameters $\varepsilon_{\text {eff }}$ and $\mu_{\text {eff }}$. We provide formulas for these two parameters; the formulas yield, in particular, that $\mu_{\text {eff }}$ can be large and that it can have a negative real part for an appropriate choice of parameters (connected to resonance).

The above description shows that we are, to some extent, following the path that was chosen in [7] (only that now the metal is perfectly conducting). The homogenization of split ring microstructures is very complex: One has to deal, at the same time, with a large contrast and with singular geometries in the homogenization setting, furthermore with three-dimensional vector calculus to perform the analysis. For these reasons, the proofs in [7] are involved.

With the contribution at hand, we analyze the case of perfectly conducting split rings. Many aspects of the analysis simplify considerably in this case. Most importantly, the magnetic cell-problem is no longer a coupled problem: in the present situation, the cell-problem can be solved with four quite explicit shape functions. Obviously, we also profit from the developments in homogenization theory of the last 10 years, e.g. the fact that we understand the system now better using the notion of geometric averaging (introduced in [4]). Neglecting the more technical parts of previous proofs, such as the description of the scattering problem, we can describe here the heart of the

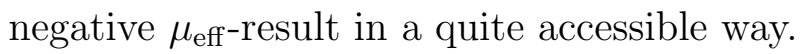

\subsection{Mathematical problem and main result}

We study the time-harmonic Maxwell equations in a complex three dimensional geometry. Let $\Omega \subset \mathbb{R}^{3}$ be a domain and let $R \subset \Omega$ be a subdomain in which many split ring resonators are distributed periodically. The metallic split rings are modelled as perfect conductors. We denote the set that is occupied by metal by $\Sigma_{\eta} \subset R$. We therefore study the system

$$
\begin{aligned}
& \operatorname{curl} E^{\eta}=i \omega \mu_{0} H^{\eta} \\
& \operatorname{curl} H^{\eta}=-i \omega \varepsilon_{0} E^{\eta}
\end{aligned}
$$

in the domain $\Omega_{\eta}=\Omega \backslash \bar{\Sigma}_{\eta}$. The number $\omega>0$ denotes the frequency, the complex numbers $\varepsilon_{0}, \mu_{0}$ are the permittivity and the permeability in vacuum. To simplify notation, we consider only the sequence $\eta_{j}=\eta_{0} 2^{-j}$.

We have to specify boundary condition on $\partial \Sigma_{\eta}$. To this end we consider trivial extensions of the fields. We denote the trivial extensions again as $E^{\eta}, H^{\eta}: \Omega \rightarrow \mathbb{C}^{3}$ (setting $E^{\eta}(x):=H^{\eta}(x):=0$ for $x \in \Sigma_{\eta}$ ). We demand that the extensions satisfy

$$
\operatorname{curl} E^{\eta}=i \omega \mu_{0} H^{\eta}
$$

in $\Omega$. Equation (1.3) provides a boundary condition: it implies that the tangential components of $E^{\eta}$ are vanishing on the boundary $\partial \Sigma_{\eta}$ (since the curl 
of $E^{\eta}$ has no singular part). We remark that (1.3) also implies that

$$
\operatorname{div} H^{\eta}=0
$$

holds in $\Omega$ for the trivial extensions. Equation (1.4) implies that the normal component of $H^{\eta}$ is vanishing on $\partial \Sigma_{\eta}$.

Theorem 1.1. Let the geometry of the problem be given by domains $\Sigma_{\eta} \subset R \subset$ $\Omega \subset \mathbb{R}^{3}$ as specified in Section 1.2. Let $\left(E^{\eta}, H^{\eta}\right)$ be a sequence of solutions of (1.1)-(1.2) with the boundary conditions expressed by (1.3). We assume that the sequence of solutions satisfies

$$
\int_{\Omega}\left|E^{\eta}\right|^{2}+\left|H^{\eta}\right|^{2} \leq C
$$

for some $C \geq 0$, independent of $\eta>0$. Let $(\hat{E}, \hat{H})$ be the geometric limit fields as defined in (1.15) and (1.16). Then there holds

$$
\begin{aligned}
\operatorname{curl} \hat{E} & =i \omega \mu_{0} \hat{\mu} \hat{H} \\
\text { curl } \hat{H} & =-i \omega \varepsilon_{0} \hat{\varepsilon} \hat{E}
\end{aligned}
$$

in the distributional sense on $\Omega$, where the effective permittivity and permeability are given as

$$
\hat{\varepsilon}(x):=\left\{\begin{array}{ll}
\varepsilon_{\mathrm{eff}} & \text { for } x \in R \\
1 & \text { for } x \in \Omega \backslash R
\end{array} \quad \hat{\mu}(x):= \begin{cases}\mu_{\mathrm{eff}} & \text { for } x \in R \\
1 & \text { for } x \in \Omega \backslash R\end{cases}\right.
$$

The tensor $\varepsilon_{\mathrm{eff}}$ is provided in (2.7) in terms of cell solutions $E^{k}(y), k=1,2,3$. The frequency dependent coefficient $\mu_{\mathrm{eff}}=\mu_{\mathrm{eff}}(\omega)$ is provided in (2.25) with the cell solutions $H^{k}(y), k=0,1,2,3$.

\subsection{Geometry}

The geometry is constructed in two steps. In the first step we describe the inclusion of the split ring in a single periodicity cell $Y$. In a second step we combine many microscopic structures to obtain a macroscopic geometry.

Microscopic geometry. We start from the periodicity cube $Y=(0,1)^{3}$; since we will always impose periodicity conditions on the cube $Y$, we may also regard it as the flat torus $\mathbb{T}^{3}$. Let a closed ring inside $Y$ be given by a set $\Sigma_{Y} \subset Y$. We assume that $\Sigma_{Y}$ is an open set with $\mathcal{C}^{2}$-boundary, not touching the boundary of $Y$, i.e. with $\bar{\Sigma}_{Y} \cap \partial(0,1)^{3}=\emptyset$. We furthermore assume that $\Sigma_{Y}$ is topologically a torus.

In order to define the split ring, we assume that $\Sigma_{Y} \backslash\left\{\left(y_{1}, y_{2}, y_{3}\right) \mid y_{1}=\right.$ $\left.0, y_{3}>0\right\}$ is an open and simply connected set. The split ring is defined as

$$
\Sigma_{Y}^{\eta}:=\Sigma_{Y} \backslash\left\{\left(y_{1}, y_{2}, y_{3}\right) \mid-\alpha \eta^{2}<y_{1}<\alpha \eta^{2}, y_{3}>0\right\}
$$


Our construction is such that the slit has the width $2 \alpha \eta^{2}$ inside the single periodicity cell. For ease of notation, we furthermore assume that the slit is a cylinder:

$$
\begin{aligned}
S_{Y}^{\eta}: & =\Sigma_{Y} \cap\left\{\left(y_{1}, y_{2}, y_{3}\right) \mid-\alpha \eta^{2}<y_{1}<\alpha \eta^{2}, y_{3}>0\right\} \\
& =\left\{\left(y_{1}, y_{2}, y_{3}\right) \mid-\alpha \eta^{2}<y_{1}<\alpha \eta^{2}, y_{3}>0,\left(0, y_{2}, y_{3}\right) \in \Sigma_{Y}\right\} .
\end{aligned}
$$

Macroscopic geometry. We study electromagnetic waves in an open set $\Omega \subset \mathbb{R}^{3}$. Contained in $\Omega$ is a second domain $R \subset \Omega$ with $\bar{R} \subset \Omega$. The set $R$ consists of meta-material, whereas on $\Omega \backslash R$ we have relative permeability and relative permittivity equal to unity.

In order to define the microstructure in $R$, we use indices $k \in \mathbb{Z}^{3}$ and shifted small cubes $Y_{k}^{\eta}:=\eta(k+Y)$. We denote by $\mathcal{K}:=\left\{k \in \mathbb{Z}^{3} \mid Y_{k}^{\eta} \subset R\right\}$ the set of indices $k$ such that the small cube $Y_{k}^{\eta}$ is contained in $R$. Here and in the following, in summations or unions over $k$, the index $k$ takes all values in the index set $\mathcal{K}$. The number of relevant indices has the order $|\mathcal{K}|=O\left(\eta^{-3}\right)$.

Using the unit-cell split rings $\Sigma_{Y}^{\eta} \subset Y$, we can now define the meta-material by setting

$$
\Sigma_{\eta}:=\bigcup_{k \in \mathcal{K}} \eta\left(k+\Sigma_{Y}^{\eta}\right) \subset R, \quad \Omega_{\eta}:=\Omega \backslash \Sigma_{\eta} .
$$

The set of slits is defined accordingly as $S_{\eta}:=\bigcup_{k \in \mathcal{K}} \eta\left(k+S_{Y}^{\eta}\right)$. The above definition of $\Omega_{\eta}$ closes the description of the system (1.1)-(1.2). Note that we do not specify boundary conditions on $\partial \Omega$; if, for example, the solution sequence $\left(E^{\eta}, H^{\eta}\right)$ satisfies a Dirichlet condition for fixed boundary data, the limit functions $(\hat{E}, \hat{H})$ will satisfy the same boundary condition.

\subsection{Two-scale limits and geometric averaging}

Our aim is to study properties of the sequence $\left(E^{\eta}, H^{\eta}\right)$ of solutions to (1.1)(1.2). We obtain these properties by characterizing limits. The most useful object is the two-scale limit of the sequence.

\section{Two-scale limits}

Since $E^{\eta}$ and $H^{\eta}$ are, by assumption, bounded in $L^{2}(\Omega)$ we can, after extraction of a subsequence, consider the two-scale limits as $\eta \rightarrow 0$ :

$$
\begin{aligned}
& E^{\eta}(x) \rightarrow E_{0}(x, y) \text { weakly in two scales, } \\
& H^{\eta}(x) \rightarrow H_{0}(x, y) \text { weakly in two scales, }
\end{aligned}
$$

for some limit functions $E_{0}, H_{0} \in L^{2}\left(\Omega \times Y, \mathbb{C}^{3}\right)$.

\section{Geometric averaging}

There are (at least) two possibilities to average a function $u: Y \rightarrow \mathbb{C}^{3}$. The standard averaging procedure is the volumetric average, given by the integral 
over $Y$. We introduce here another averaging procedure, which associates to curl-free fields $u: Y \rightarrow \mathbb{C}^{3}$ their line integrals.

In the subsequent definition, $\Sigma$ is assumed to be connected. We emphasize that we do not require $\Sigma$ to be simply connected. Actually, inclusions $\Sigma$ that are not simply connected are our motivation to modify the original definition of a geometric average (the one given in [4]).

Definition 1.2 (Geometric averaging). Let $\Sigma \subset \bar{\Sigma} \subset(0,1)^{3}$ be a connected subset of the flat torus $Y$ and let $H: Y \backslash \Sigma \rightarrow \mathbb{C}^{3}$ be a field of class $H_{\sharp}^{1}\left(Y \backslash \Sigma, \mathbb{C}^{3}\right)$ with curl $_{y} H=0$ in $Y \backslash \bar{\Sigma}$. The geometric average of $H$ is a vector $\oint H \in \mathbb{C}^{3}$ and for $k=1,2,3$, the $k$-th component of $\oint H$ is defined as a line integral over $\gamma_{k}:[0,1] \rightarrow Y, t \mapsto t e_{k}$,

$$
(\oint H)_{k}:=\int_{\gamma_{k}} H \cdot \tau
$$

where $\tau=e_{k}$ is the tangential vector of the curve $\gamma_{k}$.

We remark that, due to periodicity of $H$, the edge $\gamma_{k}$ of the torus $Y$ is inside the domain $Y \backslash \Sigma$. More important is the following observation: For every curve $\tilde{\gamma}_{k}$ that connects a point $y_{0} \in \partial(0,1)^{3}$ with $y_{0}+e_{k} \in \partial(0,1)^{3}$ and that can be obtained with an homotopy in $Y \backslash \Sigma$ from the curve $\gamma_{k}$ along the edge, the integral has the same value: $\int_{\tilde{\gamma}_{k}} H \cdot \tau=\int_{\gamma_{k}} H \cdot \tau$. This is a consequence of $\operatorname{curl}_{y} H=0$ in $Y \backslash \bar{\Sigma}$. This fact also shows that the line integral is well defined even though we only assumed $H \in H^{1}(Y \backslash \Sigma)$.

\section{Geometric limits}

Starting from $L^{2}(\Omega)$-bounded sequences $E^{\eta}$ and $H^{\eta}$ we want to define, in the appropriate sense of averaging, limit functions $\hat{E}$ and $\hat{H}$ in $L^{2}(\Omega)$.

Regarding the sequence $E^{\eta}$, we choose to define the limit $\hat{E}$ as the weak limit of $E^{\eta}$ in $L^{2}(\Omega)$,

$$
\hat{E}:=w-\lim _{\eta} E^{\eta}, \quad \hat{E}(x)=\int_{Y} E_{0}(x, y) d y .
$$

For convenience we recalled in the second equality of (1.15) a property of the two-scale limit $E_{0}$.

Instead, regarding $H^{\eta}$, we start from the two-scale limit $H_{0}(x, y)$ as defined in (1.13) and define $\hat{H}$ as the geometric average of that limit according to Definition 1.2,

$$
\hat{H}(x):=\oint H_{0}(x, .) .
$$

\section{A property of the geometric average}

The above definition of a geometric average is justified by the fact that geometric averages appear in the macroscopic equations. The underlying reason for this fact is the following property. 
Lemma 1.3 (A property of the geometric average). Let $\Sigma \subset Y$ be as in Definition 1.2. We consider $H \in H_{\sharp}^{1}\left(Y \backslash \Sigma, \mathbb{C}^{3}\right)$ with $\operatorname{curl}_{y} H=0$ in $Y \backslash \bar{\Sigma}$. On a second function $E \in H_{\sharp}^{1}\left(Y, \mathbb{C}^{3}\right)$ we assume $\operatorname{curl}_{y} E=0$ in $Y$ and $E=0$ on $\Sigma$. Then there holds

$$
\int_{Y \backslash \Sigma} H \wedge E=(\oint H) \wedge \int_{Y} E
$$

Proof. Since $E$ has vanishing curl in $Y$, we can write $E$ as a gradient of some function $\phi \in H^{1}\left((0,1)^{3}, \mathbb{C}\right)$. Since $E$ vanishes on the connected set $\Sigma$, the function $\phi$ is constant on $\Sigma$. We may assume the constant to be zero, hence

$$
E=\nabla \phi \text { in }(0,1)^{3}, \quad \phi=0 \text { in } \Sigma .
$$

We emphasize that $\phi$ is, in general, not a periodic function. We calculate the mismatch for two opposite points $y_{0}, y_{1}:=y_{0}+e_{k} \in \partial(0,1)^{3}$. Connecting the two points with a curve $\gamma$ we find

$$
\phi\left(y_{1}\right)-\phi\left(y_{0}\right)=\int_{\gamma} \nabla \phi \cdot \tau=\int_{\gamma} E \cdot \tau=\int_{Y} E \cdot e_{k},
$$

where, in the last step, we used that the integral is the same for every curve $\gamma$ due to $\operatorname{curl}_{y} E=0$ in $Y$. We obtain that the difference of the $\phi$-values on opposite faces is given by the volume average of $E$.

We can now calculate the left hand side of (1.17) with an integration by parts. Using that $\operatorname{curl}_{y} H=0$ holds in the set where $\phi \neq 0$, we find

$$
\int_{Y \backslash \Sigma} H \wedge E=\int_{(0,1)^{3}} H \wedge \nabla \phi=\int_{\partial(0,1)^{3}} H \wedge \nu \phi .
$$

We evaluate this boundary integral. For every $k \leq 3$ we consider the faces $F_{k}^{-}:=\left\{y_{k}=0\right\}$ with normal $\nu=-e_{k}$ and $F_{k}^{+}:=\left\{y_{k}=1\right\}$, with normal $\nu=e_{k}$. This yields, by periodicity of $H$,

$$
\begin{aligned}
\int_{Y \backslash \Sigma} H \wedge E & =\int_{\partial Y} H \wedge \nu \phi=\sum_{k=1}^{3}\left[\int_{F_{k}^{+}} H \wedge e_{k} \phi-\int_{F_{k}^{-}} H \wedge e_{k} \phi\right] \\
& =\sum_{k=1}^{3}\left(\int_{F_{k}^{+}} H \wedge e_{k}\right)\left(\int_{Y} E \cdot e_{k}\right) .
\end{aligned}
$$

On the face $F_{k}^{+}$, the integrand $H \wedge e_{k}$ consist of the two tangential components of $H$. The face $F_{k}^{+}$can be written as a union of straight lines. Recalling that all line integrals over tangential components of $H$ coincide because of $\operatorname{curl}_{y} H=0$, we obtain that the $F_{k}^{+}$-integral coincides with the line integrals of the geometric average (1.14). We can therefore write

$$
\int_{Y \backslash \Sigma} H \wedge E=\sum_{k=1}^{3}(\oint H) \wedge e_{k}\left(\int_{Y} E \cdot e_{k}\right)=(\oint H) \wedge \int_{Y} E .
$$

This provides (1.17) and hence the lemma. 
We note that the original definition of a geometric average in [4] was expressed in terms of formula (1.17). This definition was also used, e.g., in [21]. Our new definition is in terms of line integrals and has the advantage that more complex obstacles $\Sigma$ can be treated, e.g. obstacles where $Y \backslash \Sigma$ is not simply connected.

\section{Cell problems and effective coefficients}

It is a standard procedure to collect the equations for the two-scale limit of a sequence of solutions. In this section, we collect the cell problems for $E_{0}$ and for $H_{0}$ from (1.12)-(1.13). The important step is to find linearly independent solutions to the cell-problems such that the two-scale limits $E_{0}$ and $H_{0}$ can be written as linear combinations of these basis functions.

\subsection{Cell problem for $E_{0}$}

We investigate the two-scale limit function $E_{0}$ of (1.12). For $x \in R$ the map $Y \ni y \mapsto E_{0}(x,.) \in \mathbb{C}^{3}$ solves the following equations:

$$
\begin{aligned}
\operatorname{curl}_{y} & E_{0}=0 \text { in } Y, \\
\operatorname{div}_{y} & E_{0}=0 \text { in } Y \backslash \bar{\Sigma}_{Y}, \\
& E_{0}=0 \text { in } \Sigma_{Y}, \\
& E_{0} \text { is periodic in } Y .
\end{aligned}
$$

This set of equations follows immediately from the properties of two-scale convergence: Equation (2.1) from (1.3) and equation (2.2) from (1.2). Equation (2.3) follows from the fact that we considered trivially extended fields $E^{\eta},(2.4)$ holds always by construction of two-scale limits.

The equations can be solved with the help of three shape functions. We observe that the first equation implies that $E_{0}(x,)=.E(x)+\nabla_{y} \phi(x,$.$) , where$ $\phi$ is a scalar periodic potential in $H_{\sharp}^{1}(Y, \mathbb{C})$ and $E(x)$ denotes the average of $E_{0}(x, \cdot)$ on the unit cell. The second equation implies that $\phi$ is harmonic in $Y \backslash \bar{\Sigma}$, the third equation yields that $\phi(y)+E(x) \cdot y$ is constant on the connected subset $\Sigma$. Therefore, for a given average electric field $E(x)$, the periodic function $\phi$ is determined uniquely by the affine boundary values on $\partial \Sigma$, we demand $\phi(y)=-E(x) \cdot y$ for $y \in \Sigma$. In the above construction, there were only 3 degrees of freedom for $E_{0}$, the components of the vector $E(x)$ of the volume average. The solution space to (2.1)-(2.4) is therefore threedimensional. We denoted the volume average by $E(x)$ since the $Y$-average of the two-scale limit $E_{0}$ coincides with the weak limit $E$.

We collect our results: The two-scale limiting electric field $E_{0}(x,$.$) can be$ written as a linear combination

$$
E_{0}(x, y)=\sum_{k=1}^{3} E_{k}(x) E^{k}(y)
$$


where the real valued shape functions $E^{k}(y):=e_{k}+\nabla \phi^{k}(y)$ are given in terms of $\phi^{k}$, which is the unique solution in $H_{\sharp}^{1}(Y)$ of

$$
\Delta \phi^{k}=0 \text { in } Y \backslash \bar{\Sigma}_{Y}, \quad \phi^{k}=-y_{k} \text { on } \bar{\Sigma}_{Y} .
$$

The tensor $\varepsilon_{\text {eff }}$. By construction, the fields $E^{1}, E^{2}$, and $E^{3}$ satisfy $\int_{Y} E^{k}$. $e_{l}=\delta_{k l}$ and form a basis of the space of solutions for the $E_{0}$-cell problem. However, they are not orthonormal with respect to the usual scalar product in $L^{2}(Y)$. We define the tensor $\varepsilon_{\text {eff }}:=\left(\varepsilon_{\text {eff }}\right)_{k l} \in \mathbb{R}^{3 \times 3}$ by setting

$$
\left(\varepsilon_{\mathrm{eff}}\right)_{k l}:=\int_{Y} E^{k}(y) \cdot E^{l}(y) d y .
$$

\subsection{Cell-problem for $H_{0}$}

The magnetic field $H_{0}(x,$.$) satisfies$

$$
\begin{aligned}
\operatorname{curl}_{y} & H_{0}=0 \text { in } Y \backslash \bar{\Sigma}_{Y}, \\
\operatorname{div}_{y} & H_{0}=0 \text { in } Y, \\
& H_{0}=0 \text { in } \Sigma_{Y}, \\
& H_{0} \text { is periodic in } Y .
\end{aligned}
$$

Once more, this cell problem is an immediate consequence of two-scale convergence properties and the definition of $H_{0}$ in (1.13). Equation (2.8) follows from (1.2) and equation (2.9) follows from (1.4).

With respect to the cell-problem (2.1)-(2.4) for $E_{0}$, the role of $\operatorname{div}_{y}$ and $\operatorname{curl}_{y}$ are interchanged (in terms of the domain). This difference leads to a much richer structure of the solution space to (2.8)-(2.11): Due to the fact that $Y \backslash \Sigma_{Y}$ is not simply connected but contains one nontrivial curve, the solution space of (2.8)-(2.11) has one extra dimension - it is four-dimensional.

We derive this result in the following proposition, which is central to the analysis of the ring geometry: For closed rings, the solution space for the $H$ problem is four-dimensional. The extra dimension in the $H$-problem makes resonance effects possible and leads to the negative effective permeability for appropriate parameters.

Proposition 2.1 (The magnetic cell-problem). Let $\Sigma_{Y} \subset Y$ be as described in Section 1.2, topologically a full torus. Then the solution space to problem (2.8)-(2.11) is four-dimensional and spanned by four shape functions $H^{k}(y)$, $k=0,1,2,3$. The shape functions are uniquely determined as solutions of (2.8)-(2.11) with the following normalization: For a closed curve $\gamma_{0}:[0,1] \rightarrow$ $(0,1)^{3} \backslash \Sigma_{Y}$ with tangential vector $\tau$ which winds once through the closed ring $\Sigma_{Y}$, there holds

$$
\begin{aligned}
& \oint H^{k}=e_{k}, \quad \int_{\gamma_{0}} H^{k} \cdot \tau=0 \quad \text { for } k \in\{1,2,3\}, \text { and } \\
& \oint H^{0}=0, \quad \int_{\gamma_{0}} H^{0} \cdot \tau=1 .
\end{aligned}
$$


Proof. In this proof, which concerns only functions on the unit cube $Y$, we write $\Sigma$ instead of $\Sigma_{Y}$ for brevity.

Step 1: Construction of $H^{k}, k=1,2,3$. For fixed $k \in\{1,2,3\}$, our aim is to construct $H^{k}$ as we constructed $E^{k}$ in the lines before (2.6). We use the ansatz $H^{k}(y)=e_{k}+\nabla \phi^{k}(y)$, now $\phi^{k}$ is a periodic solution of the Neumann-problem

$$
\Delta \phi^{k}=0 \text { in } Y \backslash \bar{\Sigma}_{Y}, \quad \nu \cdot \nabla \phi^{k}=-\nu \cdot e_{k} \text { on } \partial \Sigma_{Y},
$$

where $\nu$ is the exterior normal vector field for $\Sigma$. This set of equations can be solved with $\phi^{k} \in H_{\sharp}^{1}(Y \backslash \Sigma)$ with the Lax-Milgram theorem (prescribing e.g. that the average of $\phi^{k}$ vanishes). We set $H^{k}(y)=e_{k}+\nabla \phi^{k}(y)$ for $y \in Y \backslash \bar{\Sigma}$ and $H^{k}(y)=0$ for $y \in \bar{\Sigma}$.

Let us check that $H^{k}$ is indeed a solution of the cell-problem. As a gradient field, $H^{k}$ satisfies (2.8). Since $\phi^{k}$ is harmonic, $H^{k}$ satisfies (2.9) in $Y \backslash \bar{\Sigma}$. The boundary condition for $\phi^{k}$ implies that the normal component of $H^{k}$ vanishes on $\partial \Sigma$; this provides (2.9) in $Y$. Properties (2.10) and (2.11) hold by construction.

We next determine the geometric average of $H^{k}$ by calculating line integrals. Along the curve $\gamma_{j}, j=0,1,2,3$, we obtain

$$
\int_{\gamma_{j}} H^{k} \cdot \tau=\int_{\gamma_{j}}\left(e_{k}+\nabla \phi^{k}\right) \cdot \tau=\delta_{k j} .
$$

This provides the normalization property (2.12).

Step 2: Construction of $H^{0}$. The construction of $H^{0}$ requires a refined construction, since we have to exploit the fact that the complement of the full torus is not simply connected. In a first step, we choose a smooth surface (with boundary) $D \subset Y \backslash \bar{\Sigma}$ that "closes the hole of the torus": We demand that the boundary curve $\bar{D} \cap \partial \Sigma$ is a closed curve and that $Y \backslash(\bar{\Sigma} \cup D)$ is simply connected.

For a function $\phi: Y \backslash \Sigma \rightarrow \mathbb{R}$ that possesses a trace on both sides of $D$, we denote by $[\phi]_{D}: D \rightarrow \mathbb{R}$ the jump of the function $\phi$ (we fix an arbitrary convention for the sign). We use the affine function space

$$
Z:=\left\{\phi \in H_{\sharp}^{1}(Y \backslash(\bar{\Sigma} \cup D)) \mid[\phi]_{D}=1\right\} .
$$

On $Z$, we study the minimization problem for the Dirichelet energy: Find $\phi \in Z$ such that

$$
I(\phi):=\frac{1}{2} \int_{Y \backslash \bar{\Sigma}}|\nabla \phi|^{2}=\min _{\varphi \in Z} I(\varphi) .
$$

We emphasize that we evaluate $\nabla \phi$ on the set $Y \backslash(\bar{\Sigma} \cup D)$, where the gradient exists by definition of $Z$ because of $\phi \in Z$. In the definition of $I$, we integrate the squared norm of this function (in other words: we integrate the squared norm of the regular part of $\nabla \phi)$. Minimization of the convex functional in (2.17) is possible with the direct method. 
Let $\phi^{0} \in Z$ be a solution of the minimization problem (2.17). We set $H^{0}:=\nabla \phi^{0}$ in $Y \backslash \bar{\Sigma}$ where, once more, we use only the regular part of the gradient. We extend trivially, setting $H^{0}(y)=0$ for $y \in \bar{\Sigma}$.

It remains to check the properties of $H^{0}$. For every function $\rho \in C_{\sharp}^{1}(Y \backslash \Sigma)$ and every $\varepsilon \in \mathbb{R}$, the function $\phi^{0}+\varepsilon \rho$ is contained in $Z$ (the function $\phi^{0}+\varepsilon \rho$ still has the jump 1 across $D$ ). This implies the Euler-Lagrange equation

$$
\int_{Y \backslash \bar{\Sigma}} \nabla \phi^{0} \cdot \nabla \rho=0
$$

The Euler-Lagrange equation implies, in a first step, $\Delta \phi^{0}=0$ in $Y \backslash(\bar{\Sigma} \cup D)$. In a second step (with an integration by parts), (2.18) implies the boundary condition $\nu \cdot \nabla \phi^{0}=0$ along $\partial \Sigma\left(\nu\right.$ the normal on $\partial \Sigma$ ) and $\left[\nu \cdot \nabla \phi^{0}\right]=0$ along $D$ ( $\nu$ the normal on $D)$. These properties imply that $H^{0}$ satisfies $(2.9)$ in $Y$. As a gradient, $H^{0}$ satisfies $(2.8)$ in $Y \backslash(\bar{\Sigma} \cup D)$. Equations (2.10) and (2.11) are satisfied by construction.

It remains to check (2.8) across the interface $D$. In a neighborhood of a point $y \in D$, we can consider the function $\tilde{\phi}^{0}$, defined as $\tilde{\phi}^{0}=\phi^{0}$ on one side of $D$ and $\tilde{\phi}^{0}=\phi^{0}+1$ on the other side of $D$. The construction is made such that $\tilde{\phi}^{0}$ has a vanishing jump across $D$. This fact implies that the function $\tilde{\phi}^{0}$ is of class $H^{1}$ in a neighborhood of $y$. By $(2.18), \tilde{\phi}^{0}$ is harmonic and hence locally of class $H^{2}$. This implies that the traces of $\nabla \phi^{0}=\nabla \tilde{\phi}^{0}$ have no jumps across $D$; we conclude that $H^{0}$ is locally of class $H^{1}$. This yields (2.8) in $Y \backslash \bar{\Sigma}$.

The calculation of line integrals is as in (2.15) of Step 1: Line integrals of $H^{0}$ over the curves $\gamma_{j}, j=1,2,3$, vanish by periodicity of $\phi^{0}$. Instead, the line integral over $\gamma_{0}$ is (we assume that $\gamma_{0}:[0,1] \rightarrow Y$ starts and ends in a point $y_{0} \in D$ and is entirely contained in $\left.Y \backslash(\bar{\Sigma} \cup D)\right)$

$$
\int_{\gamma_{0}} H^{0} \cdot \tau=\int_{\gamma_{0}} \nabla \phi^{0} \cdot \tau=\lim _{t \nearrow 1} \phi^{0}\left(\gamma_{0}(t)\right)-\lim _{t \searrow 0} \phi^{0}\left(\gamma_{0}(t)\right)= \pm 1
$$

Upon reversing the sign, we obtain the normalization property (2.13).

Step 3: Conclusion of the proof. We have constructed four solutions $H^{k}$, $k=0,1,2,3$, to the cell problem. The normalizations $(2.12)-(2.13)$ show that the solutions are linearly independent, hence the solution space is at least four-dimensional.

In order to show that the solution space has at most four dimensions, it suffices to show the following: If $u$ solves the cell-problem with vanishing normalization, i.e. with $\oint u=0$ and $\int_{\gamma_{0}} u \cdot \tau=0$, then $u$ necessarily vanishes.

We use the Poincaré lemma on the existence of potentials (or, better, its proof): given a curl-free function $u$, a potential $\Phi$ for $u$ can be constructed with the help of line integrals. In the domain $Y \backslash \Sigma$, the normalizations imply that, for every closed curve $\gamma$ in $Y \backslash \Sigma$, the integral $\int_{\gamma} u \cdot \tau$ vanishes. Therefore, the function $u$ has a potential, $u=\nabla \Phi$ for a periodic potential $\Phi$. Since $u$ has a vanishing divergence, $\Phi$ is harmonic. Since $u$ vanishes on $\Sigma$, the potential $\Phi$ satisfies a homogeneous Neumann condition on $\partial \Sigma$. Integration by parts 
gives $\int_{Y \backslash \Sigma} \nabla \Phi \cdot \nabla \Phi=0$ and it follows that $\Phi$ is constant. In particular, the gradient $u=\nabla \Phi$ vanishes. This shows that the four normalization conditions determine $u$ uniquely.

The fast-scale microscopic magnetic field $H_{0}(x,$.$) is a solution of the mag-$ netic cell problem. Proposition 2.1 implies that it can hence be written as a linear combination of the four cell solutions:

$$
H_{0}(x, y)=\sum_{k=0}^{3} h_{k}(x) H^{k}(y)
$$

The amplitude factor $h_{k}(x)$ tells us how much of the cell solution $H^{k}$ is present in a macroscopic point $x \in R$. The result of the slit analysis of the next section will be an expression for $h_{0}(x)$ in terms of $h_{1}(x), h_{2}(x), h_{3}(x)$.

The tensor $\mu_{\text {eff }}$. For points $x \in R$, the effective permeability is defined such that $Y$-averages can be expressed by geometric averages. More precisely, we want to define $\mu_{\text {eff }}$ such that, for $x \in R$,

$$
H(x)=\mu_{\mathrm{eff}} \cdot \hat{H}(x) .
$$

Let us investigate this relation with the help of (2.20). The weak limit is the arithmetic average of $H_{0}$, which takes the form

$$
H(x)=\int_{Y \backslash \Sigma_{Y}} H_{0}(x, y) d y=\sum_{k=0}^{3} h_{k}(x) \int_{Y \backslash \Sigma_{Y}} H^{k} .
$$

By (1.16), the geometric average is

$$
\hat{H}(x)=\oint H_{0}(x, .)=\sum_{k=0}^{3} h_{k}(x) \oint H^{k}=\left(h_{1}(x), h_{2}(x), h_{3}(x)\right) .
$$

Because of (2.21), our aim is to define the effective permeability tensor $\mu_{\text {eff }}$ such that

$$
\left(\int_{Y \backslash \Sigma_{Y}} H^{0}\right) h_{0}(x)+\sum_{k=1}^{3}\left(\int_{Y \backslash \Sigma_{Y}} H^{k}\right) h_{k}(x)=\mu_{\mathrm{eff}} \cdot\left(h_{1}, h_{2}, h_{3}\right)(x) .
$$

We anticipate the result of the next section: For constants $D_{k} \in \mathbb{R}$ we derive in (3.19) the formula

$$
h_{0}(x)=\frac{\omega^{2} \mu_{0} \varepsilon_{0}}{2 \alpha-\omega^{2} \mu_{0} \varepsilon_{0} D_{0}} \sum_{k=1}^{3} D_{k} h_{k}(x) .
$$

We demand that (2.24) holds for every vector $\left(h_{1}, h_{2}, h_{3}\right)(x)$ if $h_{0}(x)$ is given as above. This leads to the choice, for $1 \leq j, k \leq 3$,

$$
\left(\mu_{\mathrm{eff}}\right)_{j k}=\left(\int_{Y \backslash \Sigma_{Y}} H^{k}\right)_{j}+\frac{\omega^{2} \mu_{0} \varepsilon_{0}}{2 \alpha-\omega^{2} \mu_{0} \varepsilon_{0} D_{0}} D_{k}\left(\int_{Y \backslash \Sigma_{Y}} H^{0}\right)_{j} .
$$

The numbers $D_{k}$ are defined in (3.9). 


\section{Slit analysis}

The slit analysis is the core of the analysis of the split ring geometry. At this point, we make the connection between the capacitor (the slits $S_{\eta}$ ) and the inductance (the rings $\Sigma_{\eta}$ ). The result of the analysis is a relation between the strength $h_{0}$ of the special field $H^{0}$ (which points through the ring) and the other fields $H^{k}, k=1,2,3$.

On a more technical level, our analysis is based on the following idea: We calculate, in two different ways, the average of the field $E^{\eta}$ in the slit $S_{\eta}$. More precisely, we investigate, for a smooth cut-off function $\theta$, limits of the expression

$$
\int_{S_{\eta}} \frac{1}{\eta} E^{\eta}(x) \cdot e_{1} \theta(x) d x
$$

Once we have determined the limit of the expression in (3.1), we can conclude also a slightly stronger statement. To formulate this statement, we define a Radon measure $m_{\eta} \in \mathcal{M}(\Omega)$ with the help of the density of (3.1): we set

$$
m_{\eta}:=\mathbf{1}_{S_{\eta}} \frac{1}{\eta} E^{\eta} \cdot e_{1} \mathcal{L}^{3}
$$

where $\mathcal{L}^{3}$ is the three dimensional Lebesgue measure and the function $\mathbf{1}_{S_{\eta}}$ is the characteristic function of the set $S_{\eta}$. The Cauchy-Schwarz inequality implies the boundedness of

$$
\left\|m_{\eta}\right\|_{\mathcal{M}(\Omega)}=\left\|\mathbf{1}_{S_{\eta}} \frac{1}{\eta} E^{\eta}(x) \cdot e_{1}\right\|_{L^{1}(\Omega)} \leq\left(\int_{S_{\eta}}\left|E^{\eta}\right|^{2}\right)^{1 / 2}\left(\int_{S_{\eta}} \eta^{-2}\right)^{1 / 2} \leq C,
$$

where we used in the last step that the volume of $S_{\eta}$ is of order $\eta^{2}$. We can extract a subsequence $\eta \rightarrow 0$ and find a limit measure $m \in \mathcal{M}(\bar{\Omega})$ such that $m_{\eta} \rightarrow m$ weak-* in the sense of measures.

Calculating the limit of the expression in (3.1), which is nothing else than $\int_{\Omega} \theta d m_{\eta}$, we determine the distributional limit of the sequence $m_{\eta}$. Since the distributional limit and the measure limit coincide, we have thus determined the limit measure $m$. In this way, we will compute a first expression for $m$ in Section 3.1. We will then compute another formula for $m$ by a different approach in Section 3.2. Comparison of the two expressions for $m$ provides the desired formula (3.19).

\subsection{Large circles and E-equation}

In order to state the formulas for the limit measure $m$, we need the four effective quantities $D_{0}, D_{1}, D_{2}, D_{3}$. They are defined with the help of a special field $\chi: Y \rightarrow \mathbb{R}^{3}$. 


\section{Construction of test-functions}

We want to construct a field $\chi: Y \rightarrow \mathbb{R}^{3}$ that has similar character as our special $H$-field $H^{0}$; our specific requirement here is that $\operatorname{curl}_{y} \chi=e_{1}$ inside the slit of the ring.

Lemma 3.1 (The field $\chi$ ). There exists a number $\eta_{0}>0$ and a field $\chi \in$ $L^{2}\left(Y, \mathbb{R}^{3}\right)$ with

$$
\begin{aligned}
& \tau:=\operatorname{curl}_{y} \chi \in L^{2}\left(Y, \mathbb{R}^{3}\right), \\
& \tau \equiv 0 \text { in } Y \backslash \Sigma \text { and } \tau \equiv e_{1} \text { in } S_{\eta_{0}}
\end{aligned}
$$

with the normalization

$$
\int_{Y} E^{k}(y) \wedge \chi(y) d y=0
$$

for $k=1,2,3$.

We note that, by our assumption on the slits $S_{\eta}$, there holds $\tau \equiv e_{1}$ in $S_{\eta}$ for every $\eta \leq \eta_{0}$.

Proof. We start by constructing a field $\tau: Y \rightarrow \mathbb{R}^{3}$ which lives on the closed ring. For some $\eta_{0}$, we demand $\tau \equiv e_{1}$ in the slit $S_{\eta_{0}}$. We next choose an extension of $\tau$ to all of $Y$ with the properties

$$
\begin{aligned}
\tau=0 & \text { in } Y \backslash \bar{\Sigma}, \\
\operatorname{div}_{y} \tau=0 & \text { in } Y,
\end{aligned}
$$

Since the field $\tau$ has a vanishing divergence, it also has a vanishing average due to $\int_{Y} \tau \cdot e_{l}=\int_{Y} \tau \cdot \nabla y_{l}=0$. This implies that $\tau$ possesses a periodic vector potential $\chi$ with $\operatorname{curl}_{y} \chi=\tau$. The potential $\chi$ is already the desired field, it only remains to choose an appropriate average value of $\chi$ in order to satisfy the normalization (3.5).

We start by calculating the wedge-product of $\chi$ with $E^{k}$, using $E^{k}(y)=$ $e_{k}+\nabla \phi^{k}(y)$ and $\phi^{k}(y)=-y_{k}$ from (2.6):

$$
\begin{aligned}
\int_{Y} E^{k}(y) & \wedge \chi(y) d y=\int_{Y}\left(e_{k}+\nabla \phi^{k}(y)\right) \wedge \chi(y) d y \\
= & e_{k} \wedge \int_{Y} \chi(y) d y-\int_{Y} \phi^{k}(y) \operatorname{curl}_{y} \chi(y) d y \\
= & e_{k} \wedge \int_{Y} \chi(y) d y+\int_{\Sigma} y_{k} \tau(y) d y .
\end{aligned}
$$

The result contains the volume average $P$ and the weighted $\tau$-integrals $N_{k l}$ for $k, l=1,2,3$ :

$$
P:=\int_{Y} \chi(y) d y, \quad N_{k l}:=\int_{\Sigma} y_{k} \tau_{l}(y) d y .
$$


We observe that, for $1 \leq k, l \leq 3$, there holds

$$
\begin{aligned}
0 & =-\int_{Y} y_{k} y_{l} \operatorname{div}_{y} \tau(y) d y=\int_{\Sigma} \nabla\left(y_{k} y_{l}\right) \cdot \tau(y) d y \\
& =\int_{\Sigma} y_{k} \tau_{l}(y)+y_{l} \tau_{k}(y) d y=N_{k l}+N_{l k}
\end{aligned}
$$

Hence the matrix $N \in \mathbb{R}^{3 \times 3}$ is skew-symmetric. It can therefore be expressed with a vector $P \in \mathbb{R}^{3}$ such that

$$
e_{l} \cdot \int_{Y} E^{k}(y) \wedge \chi(y) d y=e_{l} \cdot\left(e_{k} \wedge P\right)-N_{k l}=0
$$

for $1 \leq k, l \leq 3$. This is the normalization condition (3.5).

Given the field $\tau$ (which provides the matrix $N$ ), we can choose a vector potential $\chi$ with the average $P$ as in (3.8). This provides the field $\chi$ with all the desired properties.

With the function $\chi$ of Lemma 3.1 and the cell solutions $H^{k}$ we define coefficients $D_{k} \in \mathbb{R}$ as

$$
D_{k}:=\int_{Y} H^{k}(y) \cdot \chi(y) d y
$$

\section{Calculation of limits}

This subsection is devoted to the first way of calculating the limit measure $m$, starting from the expression for the $E^{\eta}$-integral of (3.1). With $\chi$ of Lemma 3.1 and a cut-off function $\theta \in \mathcal{D}(R)$ we construct the function $x \mapsto \theta(x) \chi(x / \eta)$ and use it as a test-function in (1.1). Using two-scale convergence and (2.20) we obtain

$$
\begin{aligned}
\int_{\Omega} \operatorname{curl} E^{\eta}(x) \cdot \theta(x) \chi(x / \eta) d x=i \omega \mu_{0} \int_{\Omega} H^{\eta}(x) \cdot \theta(x) \chi(x / \eta) d x \\
\quad \rightarrow i \omega \mu_{0} \int_{\Omega} \sum_{k=0}^{3} h_{k}(x) \theta(x) \int_{Y} H^{k}(y) \cdot \chi(y) d y d x \\
\quad=i \omega \mu_{0} \int_{\Omega} \sum_{k=0}^{3} D_{k} h_{k}(x) \theta(x) d x .
\end{aligned}
$$

On the other hand, we can evaluate the left hand side with an integration by parts,

$$
\begin{gathered}
\int_{\Omega} \operatorname{curl} E^{\eta}(x) \cdot \theta(x) \chi(x / \eta) d x=\int_{\Omega} E^{\eta}(x) \cdot \operatorname{curl}(\theta(x) \chi(x / \eta)) d x \\
=\int_{S_{\eta}} E^{\eta} \cdot e_{1} \theta \frac{1}{\eta}+\int_{\Omega} E^{\eta} \cdot(\nabla \theta \wedge \chi(. / \eta)) .
\end{gathered}
$$


The first term is the slit integral of the $E$-field which we want to evaluate. The second term on the right hand side can be calculated using two-scale convergence: As $\eta \rightarrow 0$, there holds

$$
\int_{\Omega} E^{\eta} \cdot(\nabla \theta \wedge \chi(\cdot / \eta)) \rightarrow \sum_{k, l=1}^{3} \int_{\Omega} E_{k}(x) \partial_{l} \theta(x) \int_{Y} E^{k}(y) \cdot\left(e_{l} \wedge \chi(y)\right) d y d x
$$

The $Y$-integral vanishes by the normalization (3.5) in the construction of the field $\chi$. Our result is the following limit expression for the slit integral of (3.1): As $\eta \rightarrow 0$, there holds

$$
\int_{S_{\eta}} E^{\eta}(x) \cdot e_{1} \theta(x) \frac{1}{\eta} d x \rightarrow i \omega \mu_{0} \int_{\Omega} \sum_{k=0}^{3} D_{k} h_{k}(x) \theta(x) d x
$$

With (3.10), we have calculated the distributional limit of the measures $m_{\eta}$ of (3.2). The distributional limit coincides with the measure valued weak-* limit, we have therefore determined the limit measure $m$ :

$$
m=i \omega \mu_{0} \sum_{k=0}^{3} D_{k} h_{k}(x) d \mathcal{L}^{3}
$$

\subsection{Small circles and $H$-equation}

The aim of this section is to calculate slit integrals of (3.1) in another way. This will provide a new formula for the limit measure $m$. Comparison with (3.11) yields the desired relation on the macroscopic factors $h_{j}(x)$.

Let us sketch the idea behind this second calculation: We think of a twodimensional disk $D \subset Y$ that does not touch the split ring, $D \subset Y \backslash \Sigma_{Y}^{\eta}$. To obtain a nontrivial result, we assume that $D$ lies (partially) in the slit $S_{\eta}$; more precisely, we assume that the boundary line of $D$ is a closed curve $\Gamma \subset Y \backslash \Sigma_{Y}$, which is, topologically, a nontrivial curve in $Y \backslash \Sigma_{Y}$.

The Stokes theorem implies that the (two-dimensional) $D$-integral over $(\operatorname{curl} H) \cdot n$ (where $n$ is the normal to $D$ ) coincides with the (one-dimensional) $\Gamma$-integral over $H \cdot \tau$ (where $\tau$ is a tangential vector on $\Gamma$ ). Using equation (1.2) we obtain, loosely speaking, that the $D$-integral over $-i \omega \varepsilon_{0} E \cdot e_{1}$ coincides with the $\Gamma$-line-integral over $H$. The first is the slit integral (up to the factor $-i \omega \varepsilon_{0}$ and a factor $2 \alpha$ for the width of the slit). The latter is the amplitude of the part of $H$ that winds through the ring - essentially $h_{0}$. We recover this result by a rigorous calculation in (3.17).

\section{Construction of test-functions}

We now perform the argument rigorously with the help of test-functions. We recall that the slit $S_{Y}^{\eta}$ in the single cell $Y$ is contained in a slab $F_{Y}^{\eta}$ of width 
$2 \alpha \eta^{2}$; in formulas: $S_{Y}^{\eta} \subset F_{Y}^{\eta}:=\left\{\left(y_{1}, y_{2}, y_{3}\right) \in Y \mid-\alpha \eta^{2}<y_{1}<\alpha \eta^{2}\right\}$. We define the test function $\sigma^{\eta}: Y \rightarrow \mathbb{R}^{3}$ as follows:

$$
\sigma^{\eta}(y):= \begin{cases}0 & \text { for } y \in Y \backslash F_{Y}^{\eta} \\ e_{1} & \text { for } y \in S_{Y}^{\eta} \\ \sigma_{0}\left(y_{2}, y_{3}\right) e_{1} & \text { for } y \in F_{Y}^{\eta} \backslash S_{Y}^{\eta}\end{cases}
$$

where $\sigma_{0}$ is a smooth function $\mathbb{R}^{2} \rightarrow \mathbb{R}$. It has values 1 for arguments $\left(y_{2}, y_{3}\right)$ such that $\left(0, y_{2}, y_{3}\right) \in S_{Y}^{\eta}$, and it vanishes in a distance $\delta>0$ from this set.

By construction, the curl of $\sigma^{\eta}(y)$ vanishes outside $F_{Y}^{\eta}$ and in the slit $S_{Y}^{\eta}$. We consider the curl in an appropriate scaling,

$$
\rho^{\eta}(y):=\frac{1}{\eta^{2}} \operatorname{curl}_{y} \sigma^{\eta}(y)=\frac{1}{\eta^{2}}\left(\begin{array}{c}
0 \\
\partial_{y_{3}} \sigma_{0} \\
-\partial_{y_{2}} \sigma_{0}
\end{array}\right)\left(y_{2}, y_{3}\right) \mathbf{1}_{F_{Y}^{\eta}}(y) .
$$

We furthermore have to localize in the macroscopic variable. In this calculation, we cannot use a smooth cut-off function, but have to use a different localization argument. In the following, $P \subset R \subset \Omega$ shall be an arbitrary set that is given, for some $\eta_{0}>0$, by a union over cubes: $P=\bigcup_{k \in \mathcal{K}_{P}} \eta_{0}(k+Y)$ for some $\mathcal{K}_{P} \subset \mathbb{Z}^{n}$. In this section, the cut-off function $\theta$ is not a smooth function, but the indicator function,

$$
\theta(x):=\mathbf{1}_{P}(x)= \begin{cases}1 & x \in P \\ 0 & x \notin P .\end{cases}
$$

\section{Calculation of limits}

With $\theta$ and $\sigma^{\eta}$ as above, we use $x \mapsto \frac{1}{\eta} \theta(x) \sigma^{\eta}(x / \eta)$ as a test-function in (1.2). We obtain, on the one hand,

$$
\begin{aligned}
& \int_{\Omega} \operatorname{curl} H^{\eta}(x) \cdot \frac{1}{\eta} \theta(x) \sigma^{\eta}(x / \eta) d x=-i \omega \varepsilon_{0} \int_{\Omega} E^{\eta}(x) \cdot \frac{1}{\eta} \theta(x) \sigma^{\eta}(x / \eta) d x \\
& =-i \omega \varepsilon_{0} \int_{S_{\eta}} E^{\eta}(x) \cdot e_{1} \frac{1}{\eta} \theta(x) d x-i \omega \varepsilon_{0} \int_{\Omega \backslash S_{\eta}} E^{\eta}(x) \cdot \frac{1}{\eta} \theta(x) \sigma^{\eta}(x / \eta) d x .
\end{aligned}
$$

The first integral on the right hand side is the slit integral that we want to calculate. The other integral can be estimated by

$$
\begin{aligned}
& \left|\int_{\Omega \backslash S_{\eta}} E^{\eta}(x) \cdot \frac{1}{\eta} \theta(x) \sigma^{\eta}(x / \eta) d x\right|^{2} \\
& \quad \leq\left\|E^{\eta}\right\|_{L^{2}\left(\Omega_{\eta}\right)}^{2} \int_{\Omega \backslash S_{\eta}} \frac{1}{\eta^{2}}\left|\theta(x) \sigma^{\eta}(x / \eta)\right|^{2} d x \leq C \delta,
\end{aligned}
$$

since the support of $\sigma^{\eta}$ has a volume of order $\eta^{2}$ and a support of order $\delta \eta^{2}$ when the set $S_{\eta}$ is removed. We therefore obtain, up to errors of order $\delta$, the desired slit integral over $E^{\eta}$ on the right hand side of (3.15). 
On the other hand, we can calculate the left hand side of (3.15) with an integration by parts. We exploit the fact that $\sigma^{\eta}$ vanishes on the boundary of $Y$, whence $\sigma^{\eta}(. / \eta)$ vanishes on the boundary of the set $P$ (which is aligned with the cells for all $\left.\eta \leq \eta_{0}\right)$.

$$
\begin{aligned}
& \int_{\Omega} \operatorname{curl} H^{\eta}(x) \cdot \frac{1}{\eta} \theta(x) \sigma^{\eta}(x / \eta) d x \\
& \quad=\int_{P} H^{\eta}(x) \cdot \eta^{-2}\left(\operatorname{curl}_{y} \sigma^{\eta}\right)(x / \eta) d x=\int_{P} H^{\eta}(x) \cdot \rho^{\eta}(x / \eta) d x \\
& \quad=\int_{P} H^{\eta}(x) \cdot \rho^{\eta_{0}}(x / \eta) d x+\int_{P} H^{\eta}(x) \cdot\left(\rho^{\eta}(x / \eta)-\rho^{\eta_{0}}(x / \eta)\right) d x .
\end{aligned}
$$

In the last equality, we inserted a zero. The first integral has a limit as $\eta \rightarrow 0$ by the defining property of two-scale convergence,

$$
\int_{P} H^{\eta}(x) \cdot \rho^{\eta_{0}}(x / \eta) d x \rightarrow \int_{P} \int_{Y} H_{0}(x, y) \cdot \rho^{\eta_{0}}(y) d y d x
$$

The $Y$-integral can be evaluated by our choice of a test-function: For almost every $x \in \Omega$ holds, using integration by parts with normal vector $\nu$ on $\partial \Sigma_{Y}$ and $\operatorname{curl}_{y} H_{0}=0$,

$$
\begin{aligned}
& \int_{Y} H_{0}(x, y) \cdot \rho^{\eta_{0}}(y) d y=\int_{Y \backslash \Sigma_{Y}} H_{0}(x, y) \cdot \frac{1}{\eta_{0}^{2}} \operatorname{curl}_{y} \sigma^{\eta_{0}}(y) d y \\
& \quad=\frac{1}{\eta_{0}^{2}} \int_{F_{Y}^{\eta_{0}} \cap \partial \Sigma_{Y}} H_{0}(x, y) \cdot\left(\nu(y) \wedge e_{1}\right)=\frac{1}{\eta_{0}^{2}} \int_{F_{Y}^{\eta_{0}} \cap \partial \Sigma_{Y}} \sum_{k=0}^{3} h_{k}(x) H^{k}(y) \cdot \tau(y) \\
& =2 \alpha h_{0}(x) .
\end{aligned}
$$

In the last step we used that $\tau=\nu \wedge e_{1}$ is a tangential field along curves and the Fubini theorem to write the surface integral as an average of line integrals (the average is over $\left.y_{1} \in\left(-\alpha \eta_{0}^{2}, \alpha \eta_{0}^{2}\right)\right)$; all line integrals are, by our normalization of the functions $H^{k}(y)$, equal to $\delta_{0, k}$.

The other term on the right hand side of (3.16) is small. To show this, we write the difference $\hat{\rho}^{\eta}(y):=\rho^{\eta}(y)-\rho^{\eta_{0}}(y)$ as the curl of some vector potential $\psi_{Y}^{\eta}, \operatorname{curl}_{y} \psi_{Y}^{\eta}=\hat{\rho}^{\eta}$. Here, we use the fact that $\hat{\rho}^{\eta}$ has vanishing divergence and a vanishing flux around the torus $\Sigma$ (since $\rho^{\eta}(y)$ and $\rho^{\eta_{0}}(y)$ have identical fluxes). This implies that we can find a potential $\psi_{Y}^{\eta}$ that vanishes on the boundary $\partial(0,1)^{3}$ of the unit cell and on the boundary $\partial \Sigma_{Y}$, see Lemma A.1 in the appendix for the existence of $\psi_{Y}^{\eta}$. We claim that the potentials $\psi_{Y}^{\eta}$ are bounded in $L^{2}(Y)$. Using the estimate of Lemma A.1, it suffices to show an $H^{-1}(Y)$-bound for $\hat{\rho}^{\eta}$. The latter follows from the following calculation for a smooth test-function $\varphi: Y \rightarrow \mathbb{R}^{3}$, using $g\left(y_{2}, y_{2}\right)=\left(0, \partial_{y_{3}} \sigma_{0},-\partial_{y_{2}} \sigma_{0}\right)\left(y_{2}, y_{3}\right)$,

$$
\begin{aligned}
\left\langle\hat{\rho}^{\eta}, \varphi\right\rangle & =\int_{0}^{1} \int_{0}^{1} g\left(y_{2}, y_{3}\right) \int_{0}^{1}\left[\frac{1}{\eta^{2}} \mathbf{1}_{F_{Y}^{\eta}}(y)-\frac{1}{\eta_{0}^{2}} \mathbf{1}_{F_{Y}^{\eta_{0}}}(y)\right] \varphi(y) d y_{1} d y_{2} d y_{3} \\
& =2 \alpha \int_{0}^{1} \int_{0}^{1} g\left(y_{2}, y_{3}\right)\left[f_{-\alpha \eta^{2}}^{\alpha \eta^{2}} \varphi(y) d y_{1}-f_{-\alpha \eta_{0}^{2}}^{\alpha \eta_{0}^{2}} \varphi(y) d y_{1}\right] d y_{2} d y_{3},
\end{aligned}
$$


hence

$$
\left|\left\langle\hat{\rho}^{\eta}, \varphi\right\rangle\right| \leq 2 \alpha \int_{0}^{1} \int_{0}^{1} g\left(y_{2}, y_{3}\right) \int_{-\alpha \eta_{0}^{2}}^{\alpha \eta_{0}^{2}}\left|\partial_{y_{1}} \varphi(y)\right| d y_{1} d y_{2} d y_{3} \leq C\|\varphi\|_{H^{1}(Y)}
$$

This calculation shows that the potentials $\psi_{Y}^{\eta}$ are bounded in $L^{2}(Y)$.

Using the potential $\psi_{Y}^{\eta}$ and the support $P_{\eta} \subset P$ of the function $P \ni x \mapsto$ $\psi_{Y}^{\eta}(x / \eta)$ we can calculate

$$
\begin{gathered}
\int_{P} H^{\eta}(x) \cdot\left(\rho^{\eta}(x / \eta)-\rho^{\eta_{0}}(x / \eta)\right) d x=\int_{P_{\eta}} H^{\eta}(x) \cdot \operatorname{curl}_{y} \psi_{Y}^{\eta}(x / \eta) d x \\
=\eta \int_{P} \operatorname{curl}_{x} H^{\eta}(x) \cdot \psi_{Y}^{\eta}(x / \eta) d x \rightarrow 0
\end{gathered}
$$

by $L^{2}\left(\Omega_{\eta}\right)$-boundedness of $\operatorname{curl}_{x} H^{\eta}(x)$ and $\psi_{Y}^{\eta}(. / \eta)$.

Collecting the results from (3.15) and (3.16) we find for the limit $\eta \rightarrow 0$

$$
m_{\eta}(P)=\int_{S_{\eta}} E^{\eta}(x) \cdot e_{1} \frac{1}{\eta} \theta(x) d x \rightarrow \frac{2 \alpha}{-i \omega \varepsilon_{0}} \int_{P} h_{0}(x) d x+O(\delta) .
$$

The limit of the left hand side exists by choice of the subsequence $\eta$, the limit is given by $m(P)$. Since $\delta$ was arbitrary, (3.17) gives the desired second relation for $m$.

\section{Result of the slit analysis}

It remains to compare the two expression for $m$ obtained in (3.17) and in (3.11). We obtain

$$
\frac{2 \alpha}{-i \omega \varepsilon_{0}} h_{0}(x)=i \omega \mu_{0} \sum_{k=0}^{3} D_{k} h_{k}(x) \text {. }
$$

This provides a frequency-dependent formula for $h_{0}$ in terms of $h_{1}, h_{2}, h_{3}$ :

$$
h_{0}(x)=\frac{\omega^{2} \mu_{0} \varepsilon_{0}}{2 \alpha-\omega^{2} \mu_{0} \varepsilon_{0} D_{0}} \sum_{k=1}^{3} D_{k} h_{k}(x) .
$$

\section{Macroscopic constitutive laws}

In the subsequent calculations we use, additionally to the geometric limits $\hat{E}$ and $\hat{H}$, also the weak limits $E^{\eta} \rightarrow E=\hat{E}$ and $H^{\eta} \rightarrow H$ in $L^{2}(\Omega)$. 
Limit process in (1.1). We can take the distributional limit of (1.1) (or, more precisely, since we consider the trivial extensions, the distributional limit of equation (1.3)). We obtain, in the limit $\eta \rightarrow 0$,

$$
\operatorname{curl} \hat{E} \leftarrow \operatorname{curl} E^{\eta}=i \omega \mu_{0} H^{\eta} \rightarrow i \omega \mu_{0} H=i \omega \mu_{0} \hat{\mu} \hat{H} .
$$

We recall that the last equation is a consequence of the definition of $\hat{\mu}$ and $\hat{H}$, see (2.21) together with $\hat{\mu}=\mu_{\text {eff }}$ in $R$ and $\hat{\mu}=1$ in $\Omega \backslash R$. The above distributional limit equation already provides (1.6), the first of the two effective equations.

Limit process in (1.2). We use an oscillating test-function. We choose a smooth function $\theta: \Omega \rightarrow \mathbb{R}$ with compact support and fix $j \in\{1,2,3\}$. We consider $\psi_{\eta}(x)=E_{\eta}^{j}(x) \theta(x)$ with $E_{\eta}^{j}(x)=E^{j}(x / \eta)$. The second Maxwell equation (1.2) yields

$$
\int_{\Omega} \operatorname{curl} H^{\eta} \cdot \psi_{\eta}=-i \omega \varepsilon_{0} \int_{\Omega} E^{\eta} \cdot \psi_{\eta}
$$

It remains to evaluate the limits of both sides of (4.2). We start with the left hand side. In the subsequent calculation we use first integration by parts and $\operatorname{curl}_{y} E^{j}=0$, then the property of two-scale convergence.

$$
\begin{gathered}
\int_{\Omega} \operatorname{curl} H^{\eta} \cdot \psi_{\eta}=\int_{\Omega} H^{\eta} \cdot \operatorname{curl} \psi_{\eta}=-\int_{\Omega} H^{\eta}(x) \cdot\left(E_{\eta}^{j}(x) \wedge \nabla \theta(x)\right) d x \\
\left.\rightarrow-\int_{\Omega} \int_{Y}\left(H_{0}(x, y) \wedge E^{j}(y)\right) \cdot \nabla \theta(x)\right) d y d x \\
\stackrel{(1.17)}{=}-\int_{\Omega}\left(\oint H_{0}(x, .)\right) \wedge\left(\int_{Y} E^{j}\right) \cdot \nabla \theta(x) d x \\
\stackrel{(1.16)}{=}-\int_{\Omega} \hat{H}(x) \wedge e_{j} \cdot \nabla \theta(x) d x=\int_{\Omega}(\operatorname{curl} \hat{H}) \cdot e_{j} \theta .
\end{gathered}
$$

We now calculate the limit of the right hand side of (4.2). In the first equality, we use that $E_{\eta}^{j}(x / \eta)$ vanishes on $\Sigma_{\eta} \cup S_{\eta}$, in the second we use twoscale convergence. In the last equation we use the definition of $\varepsilon_{\text {eff }}$ and the fact that $\hat{\varepsilon}$ from (1.8) coincides with $\varepsilon_{\text {eff }}$ in $R$.

$$
\begin{aligned}
\int_{\Omega} E^{\eta} \cdot \psi_{\eta} & =\int_{\Omega \backslash\left(\Sigma_{\eta} \cup S_{\eta}\right)} E^{\eta}(x) \cdot E_{\eta}^{j}(x) \theta(x) d x \\
& \rightarrow \int_{\Omega} \int_{Y} E_{0}(x, y) \cdot E^{j}(y) \theta(x) d y d x \stackrel{(2.7)}{=} \int_{\Omega} \hat{\varepsilon} E(x) \cdot e_{j} \theta(x) d x .
\end{aligned}
$$

Since $j \in\{1,2,3\}$ and $\theta=\theta(x)$ are arbitrary, (4.2) implies

$$
\operatorname{curl} \hat{H}(x)=-i \omega \varepsilon_{0} \hat{\varepsilon}(x) E(x)
$$

for almost every $x \in \Omega$. Because of $\hat{E}=E$, we have obtained the effective equation (1.7). This concludes the proof of the main theorem. 


\section{A Construction of vector potentials}

In the slit analysis, we made use of the following Lemma. The lemma is applied with the domain $R$ that is not $\Sigma$, but a torus that lies in the complement $Y \backslash \Sigma$ and that winds around the torus $\Sigma$.

The essential part of this lemma can be found as Theorem 3.17 of [2]. Since very general domains are studied in [2], we have chosen to provide a sketch of proof here.

Lemma A.1 (Construction of vector potentials in tori). Let $R \subset \bar{R} \subset(0,1)^{3}=$ $Y$ be an open subset which is, topologically, a torus. Let $D \subset R$ be a twodimensional surface such that $R \backslash D$ is simply connected. We assume that $\partial R$ and $D$ are of class $\mathcal{C}^{2}$ and denote a normal vector field on $D$ by $n$ and a normal vector field on $\partial R$ by $\nu$. Let $\rho: R \rightarrow \mathbb{R}^{3}$ be a vector field of class $L^{2}(R)$ with the properties

$$
\begin{aligned}
\operatorname{div} \rho=0 & \text { in } R, \\
\rho \cdot \nu & =0 \quad \text { on } \partial R, \\
\int_{D} \rho \cdot n & =0 .
\end{aligned}
$$

Then there exists a vector potential $\psi: R \rightarrow \mathbb{R}^{3}$ satisfying

$$
\begin{aligned}
\operatorname{curl} \psi=\rho & \text { in } R, \\
\operatorname{div} \psi=0 & \text { in } R, \\
\psi \wedge \nu=0 & \text { on } \partial R .
\end{aligned}
$$

For a constant $C \geq 0$ that depends on $R$, but not on $\rho$, there holds

$$
\|\psi\|_{L^{2}(R)} \leq C\|\rho\|_{H^{-1}(R)} .
$$

Proof. Construction of the potential. We consider the space

$$
X:=\left\{\psi \in H^{1}\left(R, \mathbb{R}^{3}\right) \mid \psi \wedge \nu=0 \text { on } \partial R\right\}
$$

and the bilinear form $a: X \times X \rightarrow \mathbb{R}$,

$$
a(\psi, \varphi):=\int_{R} \operatorname{curl} \psi \cdot \operatorname{curl} \varphi+\int_{R} \operatorname{div} \psi \operatorname{div} \varphi
$$

We start with an investigation of the kernel of the corresponding operator: Let $\psi \in X$ be a function with $a(\psi, \psi)=0$. Then $\operatorname{div} \psi=0$ and $\operatorname{curl} \psi=0$ hold by definition of $a$. As a curl-free function, $\psi$ is locally the gradient of a harmonic function $\Phi$. The boundary condition in the space $X$ implies that all integrals over $\psi$ over closed curves vanish, hence $\Phi$ is a global potential. The definition of $X$ furthermore yields that $\Phi$ is constant on $\partial R$. We find that $\Phi$ is constant in $R$ and $\psi \equiv 0$. We conclude that $a$ has a trivial kernel. 
The bilinear form $a$ is coercive on $X$, see e.g. [13] for this classical result. We must be very careful in this statement: In [13], coerciveness is understood in the sense that $a(\psi, \psi)+\|\psi\|_{L^{2}}^{2}$ is equivalent to the squared $H^{1}$-norm. But in our case (namely when the bilinear form has a trivial kernel), the coercivity of $a$ can be concluded also in the classical sense, i.e. $\|\psi\|_{H^{1}}^{2} \leq C a(\psi, \psi)$ for all $\psi \in X$, compare [26].

Coercivity of $a$ allows to invoke the Lax-Milgram theorem. For $\rho$ as in the lemma, we find a solution $\psi \in X$ of the problem

$$
a(\psi, \varphi)=\int_{R} \rho \cdot \operatorname{curl} \varphi \quad \forall \varphi \in X .
$$

We observe the following: An arbitrary scalar $L^{2}(R)$-function $h: R \rightarrow \mathbb{R}$ can be written as $h=\operatorname{div} \varphi$ for some $\varphi \in X$ with $\operatorname{curl} \varphi=0$; indeed, we can choose $\varphi=\nabla \phi$ and solve the Poisson problem for $\Delta \phi=h$ with $\phi=0$ on $\partial R$. Inserting $\varphi$ in (A.10) shows that the solution $\psi$ has a vanishing divergence.

It remains to collect the equations that are satisfied by the difference $u:=$ curl $\psi-\rho$. The divergence of $u$ vanishes by assumption (A.1) on $\rho$. The curl of $u$ vanishes in $R$ by (A.10). The normal component of $u$ vanishes on $\partial R$, since $\psi \in X$ has no tangential components on $\partial R$ and since the normal component of $\rho$ vanishes on $\partial R$. Finally, $u$ also satisfies the normalization (A.3): $\rho$ satisfies it by assumption and curl $\psi$ satisfies it by Stokes theorem. We conclude that $u$ can be written as the gradient of a harmonic function on $R$ (the normalization (A.3) allows to extend a local potential to a global potential). The potential is harmonic and satisfies a homogeneous Neumann condition, hence it is constant and $u$ vanishes. This provides (A.4). Property (A.6) holds by construction of $\psi \in X$ and (A.5) has been shown before.

Estimate for the potential. We use that $\psi$ posesses again a vector potential. This statement (with the correct boundary conditions for $\psi$ ) appears, e.g., as Theorem 3.12 in [2], but the proof can also be developed along the above lines: We write $\psi=\operatorname{curl} \Phi$ with $\operatorname{div} \Phi=0$ in $R$ and $\Phi \cdot \nu=0$ on $\partial R$ and the normalization (A.3). The potential $\Phi$ solves $\Delta \Phi=\rho$ and is therefore controlled in $H^{1}(R)$ by the $H^{-1}(R)$-norm of $\rho$. This implies the estimate (A.7).

\section{Acknowledgements}

Support of the second author by DFG grant Schw 639/6-1 is gratefully acknowledged.

\section{References}

[1] G. Allaire. Homogenization and two-scale convergence. SIAM J. Math. Anal., 23(6):1482-1518, 1992.

[2] C. Amrouche, C. Bernardi, M. Dauge, and V. Girault. Vector potentials in three-dimensional non-smooth domains. Math. Methods Appl. Sci., 21(9):823-864, 1998. 
[3] A. Bensoussan, J.-L. Lions, and G. C. Papanicolaou. Homogenization in deterministic and stochastic problems. In Stochastic problems in dynamics (Sympos., Univ. Southampton, Southampton, 1976), pages 106-115. Pitman, London, 1977.

[4] G. Bouchitté, C. Bourel, and D. Felbacq. Homogenization of the 3D Maxwell system near resonances and artificial magnetism. C. R. Math. Acad. Sci. Paris, 347(9-10):571-576, 2009.

[5] G. Bouchitté and D. Felbacq. Homogenization near resonances and artificial magnetism from dielectrics. C. R. Math. Acad. Sci. Paris, 339(5):377$382,2004$.

[6] G. Bouchitté and D. Felbacq. Homogenization of a wire photonic crystal: the case of small volume fraction. SIAM J. Appl. Math., 66(6):2061-2084, 2006.

[7] G. Bouchitté and B. Schweizer. Homogenization of Maxwell's equations in a split ring geometry. Multiscale Model. Simul., 8(3):717-750, 2010.

[8] G. Bouchitté and B. Schweizer. Plasmonic waves allow perfect transmission through sub-wavelength metallic gratings. Netw. Heterog. Media, 8(4):857-878, 2013.

[9] Y. Chen and R. Lipton. Tunable double negative band structure from non-magnetic coated rods. New Journal of Physics, 12(8):083010, 2010.

[10] Y. Chen and R. Lipton. Double negative dispersion relations from coated plasmonic rods. Multiscale Modeling and Simulation, 209(3):835-868, 2013.

[11] Y. Chen and R. Lipton. Multiscale methods for engineering double negative metamaterials. Photonics and Nanostructures Fundamentals and Applications, 11(4):442-454, 2013.

[12] Y. Chen and R. Lipton. Resonance and double negative behavior in metamaterials. Arch. Ration. Mech. Anal., 11(1):192-212, 2013.

[13] M. Costabel. A coercive bilinear form for Maxwell's equations. J. Math. Anal. Appl., 157(2):527-541, 1991.

[14] D. Felbacq and G. Bouchitté. Homogenization of a set of parallel fibres. Waves Random Media, 7(2):245-256, 1997.

[15] V. Jikov, S. Kozlov, and O. Oleinik. Homogenization of Differential Operators and Integral Functionals. Springer, 1994.

[16] M. Kafesaki, I. Tsiapa, N. Katsarakis, Th. Koschny, C. M. Soukoulis, and E. N. Economou. Left-handed metamaterials: The fishnet structure and its variations. Physical Review, B 75:235114-1-235114-9, 2007. 
[17] R. V. Kohn, J. Lu, B. Schweizer, and M. I. Weinstein. A variational perspective on cloaking by anomalous localized resonance. Comm. Math. Phys., 328(1):1-27, 2014.

[18] R.V. Kohn and S. Shipman. Magnetism and homogenization of microresonators. Multiscale Modeling \&6 Simulation, 7(1):62-92, 2007.

[19] A. Lamacz and B. Schweizer. Effective Maxwell equations in a geometry with flat rings of arbitrary shape. SIAM J. Math. Anal., 45(3):1460-1494, 2013.

[20] A. Lamacz and B. Schweizer. Effective acoustic properties of a metamaterial consisting of small Helmholtz resonators. DCDS-S, 2016.

[21] A. Lamacz and B. Schweizer. A negative index meta-material for Maxwells equations. SIAM J. Math. Anal., 2016. (in press).

[22] G. W. Milton and N.-A. P. Nicorovici. On the cloaking effects associated with anomalous localized resonance. Proc. R. Soc. Lond. Ser. A Math. Phys. Eng. Sci., 462:3027-3059, 2006.

[23] A. B. Movchan and S. Guenneau. Split-ring resonators and localized modes. Phys. Rev. B, 70(12):125116, 2004.

[24] J.B. Pendry. Negative refraction makes a perfect lens. Phys. Rev. Lett., 85(3966), 2000.

[25] J.B. Pendry, D. Schurig, and D.R. Smith. Controlling electromagnetic fields. Science, 312(5781), 2006.

[26] B. Schweizer. Friedrichs inequality, Helmholtz decomposition, vector potentials, and the div-curl lemma. Technical report, TU Dortmund, 2016. To appear in INDAM-Springer series.

[27] B. Schweizer. Resonance meets homogenization - construction of metamaterials with astonishing properties. Jahresberichte der DMV, 2016. DOI: $10.1365 / \mathrm{s} 13291-016-0153-2$.

[28] A. Sellier, S. N. Burokur, B. Kanté, and A. de Lustrac. Novel cut wires metamaterial exhibiting negative refractive index. IEEE, 2009.

[29] R. A. Shelby, D. R. Smith, and S. Schultz. Experimental verification of a negative index of refraction. Science, 292(5514):77-79, 2001.

[30] V.G. Veselago. The electrodynamics of substances with simultaneously negative values of $\varepsilon$ and $\mu$. Soviet Physics Uspekhi, 10:509-514, 1968. 


\section{Preprints ab 2013/09}

2016-05 Robert Lipton and Ben Schweizer

Effective Maxwell's equations for perfectly conducting split ring resonators

2016-04 Ben Schweizer

Resonance meets homogenization - Construction of meta-materials with astonishing properties

2016-03 Ben Schweizer

On Friedrichs inequality, Helmholtz decomposition, vector potentials, and the div-curl lemma

2016-02 Michael Voit

Generalized commutative association schemes, hypergroups, and positive product formulas

2016-01 Agnes Lamacz and Ben Schweizer

Effective acoustic properties of a meta-material consisting of small Helmholtz resonators

2015-13 Christian Eggert, Ralf Gäer, Frank Klinker

The general treatment of non-symmetric, non-balanced star circuits: On the geometrization of problems in electrical metrology

2015-12 Daniel Kobe and Jeannette H.C. Woerner

Oscillating Ornstein-Uhlenbeck processes and modelling electricity prices

2015-11 Sven Glaser

A distributional limit theorem for the realized power variation of linear fractional stable motions

2015-10 Herold Dehling, Brice Franke and Jeannette H.C. Woerner

Estimating drift parameters in a fractional Ornstein Uhlenbeck process with periodic mean

2015-09 Harald Garcke, Johannes Kampmann, Andreas Rätz and Matthias Röger

A coupled surface-Cahn-Hilliard bulk-diffusion system modeling lipid raft formation in cell membrans

2015-08 Agnes Lamacz and Ben Schweizer

Outgoing wave conditions in photonic crystals and transmission properties at interfaces

2015-07 Manh Hong Duong, Agnes Lamacz, Mark A. Peletier and Upanshu Sharma

Variational approach to coarse-graining of generalized gradient flows

2015-06 Agnes Lamacz and Ben Schweizer

A negative index meta-material for Maxwell's equations

2015-05 Michael Voit

Dispersion and limit theorems for random walks associated with hypergeometric functions of type $B C$

2015-04 Andreas Rätz

Diffuse-interface approximations of osmosis free boundary problems

2015-03 Margit Rösler and Michael Voit

A multivariate version of the disk convolution

2015-02 Christina Dörlemann, Martin Heida, Ben Schweizer

Transmission conditions for the Helmholtz-equation in perforated domains 
Program of the International Conference

Geometric and Algebraic Methods in Mathematical Physics

March 16-19, 2015, Dortmund

2014-10 Frank Klinker

An explicit description of SL $(2, \mathbb{C})$ in terms of $\mathrm{SO}^{+}(3,1)$ and vice versa

2014-09 Margit Rösler and Michael Voit

Integral representation and sharp asymptotic results for some Heckman-Opdam hypergeometric functions of type $\mathrm{BC}$

2014-08 Martin Heida and Ben Schweizer

Stochastic homogenization of plasticity equations

2014-07 Margit Rösler and Michael Voit

A central limit theorem for random walks on the dual of a compact Grassmannian

2014-06 Frank Klinker

Eleven-dimensional symmetric supergravity backgrounds, their geometric superalgebras, and a common reduction

2014-05 Tomáš Dohnal and Hannes Uecker

Bifurcation of nonlinear Bloch waves from the spectrum in the Gross-Pitaevskii equation

2014-04 Frank Klinker

A family of non-restricted $D=11$ geometric supersymmetries

2014-03 Martin Heida and Ben Schweizer

Non-periodic homogenization of infinitesimal strain plasticity equations

2014-02 Ben Schweizer

The low frequency spectrum of small Helmholtz resonators

2014-01 Tomáš Dohnal, Agnes Lamacz, Ben Schweizer

Dispersive homogenized models and coefficient formulas for waves in general periodic media

2013-16 Karl Friedrich Siburg

Almost opposite regression dependence in bivariate distributions

2013-15 Christian Palmes and Jeannette H. C. Woerner

The Gumbel test and jumps in the volatility process

2013-14 Karl Friedrich Siburg, Katharina Stehling, Pavel A. Stoimenov, Jeannette H. C. Wörner

An order for asymmetry in copulas, and implications for risk management

2013-13 Michael Voit

Product formulas for a two-parameter family of Heckman-Opdam hypergeometric functions of type BC

2013-12 Ben Schweizer and Marco Veneroni

Homogenization of plasticity equations with two-scale convergence methods

2013-11 Sven Glaser

A law of large numbers for the power variation of fractional Lévy processes

2013-10 Christian Palmes and Jeannette H. C. Woerner

The Gumbel test for jumps in stochastic volatility models

2013-09 Agnes Lamacz, Stefan Neukamm and Felix Otto

Moment bounds for the corrector in stochastic homogenization of a percolation model 
